\title{
Effect of respiratory syncytial virus on the growth of hepatocellular carcinoma cell-lines
}

\author{
Song Hee Choi ${ }^{1}$, Byoung Kwon Park ${ }^{1}$, Keun-Wook Lee ${ }^{2}$, Jun Chang ${ }^{3}$, Younghee Lee ${ }^{4}$ E Hyung-Joo Kwon ${ }^{1,5, *}$ \\ Departments of ${ }^{1}$ Microbiology, College of Medicine, ${ }^{2}$ Biomedical Science, College of Natural Science, Hallym University, Chuncheon \\ 24252, ${ }^{3}$ Graduate School of Pharmaceutical Sciences, Ewha Womans University, Seoul 03760, ${ }^{4}$ Department of Biochemistry, College of \\ Natural Sciences, Chungbuk National University, Cheongju 28644, ${ }^{5}$ Center for Medical Science Research, College of Medicine, Hallym \\ University, Chuncheon 24252, Korea
}

In several reports, the respiratory syncytial virus (RSV) was identified as an oncolytic virus in cancer cells (e.g., lung and prostate cancer). However, the effects of RSV in hepatocellular carcinoma (HCC) cells have not yet been investigated. Here, we observed the inhibitory effects of RSV infection in HCC cell-lines. Cell growth was significantly decreased by RSV infection in BNL-HCC, Hep3B, Huh-7 and SNU-739 cells. After RSV infection, plaque formation and syncytial formation were observed in affected Hep3B and Huh-7 cells. RSV protein-expression was also detected in Hep3B and Huh-7 cells; however, only Huh-7 cells showed apoptosis after RSV infection. Furthermore, inhibition of cell migration by RSV infection was observed in BNL-HCC, Hep3B, Huh-7 and SNU-739 cells. Therefore, further investigation is required to clarify the molecular mechanism of RSV-mediated inhibition of HCC cell growth, and to develop potential RSV oncolytic viro-therapeutics. [BMB Reports 2015; 48(10): 565-570]

\section{INTRODUCTION}

Cancer is currently a major cause of death worldwide. To develop prophylactic vaccines and therapeutics against cancer, diverse trials have been undertaken. Radiotherapy, chemotherapy, and immunotherapy have been developed conventionally, but the prevention of, and effective therapy for cancer is still problematic.

Hepatocellular carcinoma (HCC) is one of the most common cancers in the world, and causes more than 500,000 deaths per year (1-3). The frequency of HCC is higher in Asia and Africa, but the occurrence of HCC is increasing most quickly in the United

${ }^{*}$ Corresponding author. Tel: +82-33-248-2635; Fax: +82-33-2413640; E-mail: hjookwon@hallym.ac.kr

http://dx.doi.org/10.5483/BMBRep.2015.48.10.268

Received 22 December 2014, Revised 14 January 2015, Accepted 5 March 2015

Keywords: Apoptosis, Cell growth, Hepatocellular carcinoma, Oncolytic effect, RSV
States (4). It is known that the emergence of HCC is initiated by hepatitis virus infection or liver cirrhosis. Two types of hepatitis virus (i.e., hepatitis $B$ and hepatitis $C$ ) are involved in the incidence of HCC (5). Conventional chemotherapy and radiotherapy against HCC have been developed, but so far exhibit limited efficacy $(6,7)$. Immunotherapy was also investigated in an attempt to improve the limited efficacy of HCC therapy (8).

To improve cancer therapy, many researchers have investigated other therapy systems such as oncolytic viro-therapy $(9$, 10). One example of an oncolytic immunotherapeutic virus in HCC was reported: a vaccinia virus, named JX-594 (Pex-Vec). JX-594 is the vaccinia virus genetically modified to improve cancer selectivity, immune stimulation, and replication assessment (11-13). Injection of JX-594 in HCC patients induced the replication of JX-594 viral genome, expression of granulocyte-macrophage colony-stimulating factor, and anticancer immunity (14).

Human respiratory syncytial virus (RSV) is an enveloped, non-segmented negative-sense single-stranded RNA virus belonging to the paramyxovirus family $(15,16)$. Transcription and replication of the RSV RNA genome occurs in the cytoplasm without nuclear association. The RSV is a lung pathogen involving lower respiratory tract infection in infants and immunocompromised adults $(17,18)$. RSV infection is associated with diverse respiratory symptoms such as mild common colds, bronchiolitis, and pneumonia. Recently, oncolytic characteristics of RSV were reported in lung, skin, and prostate cancer cells (19-21). The oncolytic effect of the RSV was detected in human prostate cancer cells including PC-3, LNCaP, and RM1, during in vitro experiments and in a xenograft mouse model $(19,20)$. Furthermore, RSV-induced cytotoxic effect and apoptosis was reported in the human skin-cancer cell-line, A431 (21).

Here, we observed growth inhibition induced by RSV infection in HCC cell lines. We also analyzed the anti-migratory function and cell-cycle-arrest properties of RSV in vitro in HCC cells.

\section{RESULTS}

Inhibition of cell growth in cancer cell lines after RSV infection

To measure the effect of RSV infection on the growth of HCC

ISSN: 1976-670X (electronic edition)

Copyright (C) 2015 by the The Korean Society for Biochemistry and Molecular Biology

(c) This is an open-access article distributed under the terms of the Creative Commons Attribution Non-Commercial License (http://creativecommons.org/licenses/by-nc/4.0) which permits unrestricted non-commercial use, distribution, and reproduction in any medium, provided the original work is properly cited. 
and colon-cancer cell lines, cells were infected with RSV A2 strain and the growth of cells was analyzed by MTT assay. The growth of BNL-HCC, Hep3B, Huh-7 and SNU-739 cells was significantly decreased, depending on the time course of RSV infection at the $\mathrm{MOI}$ of 0.1 (Fig. 1A, B, C and D). Cell growth, in particular, was dramatically decreased five days after infection. However, the growth of other cells (i.e., SNU-761 and SNU-423) did not change after RSV infection (data not shown). In the case of colon cancer cell lines (CT-26, HCT-116, HT-29 and LoVo), the growth was not significantly affected by RSV at the $\mathrm{MOI}$ of 0.1 up to 5 days (Fig. 1E, F, G and H). Colon cancer cells don't grow at 5 days after plating, therefore there is no effect. In addition, we performed supplementary experiments with $\mathrm{MOI}$ of 0.01 and 1 to find optimal virus titer for treatment. However, there was no significant change except HT-29. In the case of HT-29 cells, the cell growth was decreased about $25 \%$ five days after infection of $1 \mathrm{MOI}$ RSV. These results demonstrate that the growth of BNL-HCC, Hep3B, Huh-7, and SNU-739 cells is influenced by RSV infection.

\section{Plaque formation and cell morphology changes after RSV infection}

It is not clear whether RSV can infect HCC cell-lines and colon-cancer cell-lines. Therefore, we tested susceptibility of HCC and colon cancer cells to RSV infection using a plaque assay. The cytopathogenic effects and plaque formation was detected five days post infection in Hep3B, Huh-7, and CT-26 cells. However, such effects were not detected in the other cells tested (Fig. 2A-C). In contrast to the results in Fig. 1, plaque formation was not found in BNL-HCC and SNU-739 cells after RSV infection (Fig. 2C).

Cells form a syncytium, a so-called giant cell, after RSV infection (17). Therefore, RSV-infected cancer cells were stained with Giemsa solution to observe the syncytial formation. Hep3B and Huh-7 cells showed syncytial formation after RSV infection, but others (BNL-HCC and SNU-739) did not (Fig. 2D).

To investigate the expression of RSV proteins in cells three days post infection, HCC cells were stained with anti-RSV antibody and analyzed by confocal microscopy. As shown in Fig. 3A, Hep3B and Huh-7 cell lines showed the RSV protein expression on the cell surface and in cytosol. These results suggest that decreased growth of Hep3B and Huh-7 cells is caused by RSV infection and replication. However, RSV protein expression was not detected in BNL-HCC and SNU-739 cell lines (Fig. 3A). As the sensitivity of immunofluorescence staining and confocal microscopy is limited depending on the reactivity of antibody and the target protein amount, we checked expression of RSV proteins by Western blot analysis using a specific antibody to RSV G protein as an example. As shown in Fig. 3B, low level of $\mathrm{G}$ protein expression was detected in BNL-HCC and SNU-739 cell lines. Therefore, it is likely that a different mechanism is operating in the growth inhibition by RSV infection in BNL-HCC and SNU-739 cells.

\section{Inhibition of cell migration after RSV infection}

To investigate the effect of RSV infection on the migration properties of cancer cells, RSV-infected cells were loaded on top of a trans-well chamber and migration assays were performed. As shown in Fig. 4A and 4B, migrations of all the tested HCC cell lines were inhibited by RSV infection. However, the migration of other cells (i.e., SNU-761 and SNU-423) did not influenced by RSV infection (data not shown).

\section{Apoptosis in Huh-7 cells after RSV infection}

A previous report showed that apoptosis was induced by RSV infection in prostate cancer cells (20). To investigate whether apoptosis is induced by RSV infection in HCC cells, a TUNEL assay was performed. As shown in Fig. 4B, Huh-7 cells showed apoptosis after RSV infection. However, there was no apoptosis after RSV infection in BNL-HCC (Fig. 4C), Hep3B and SNU-739
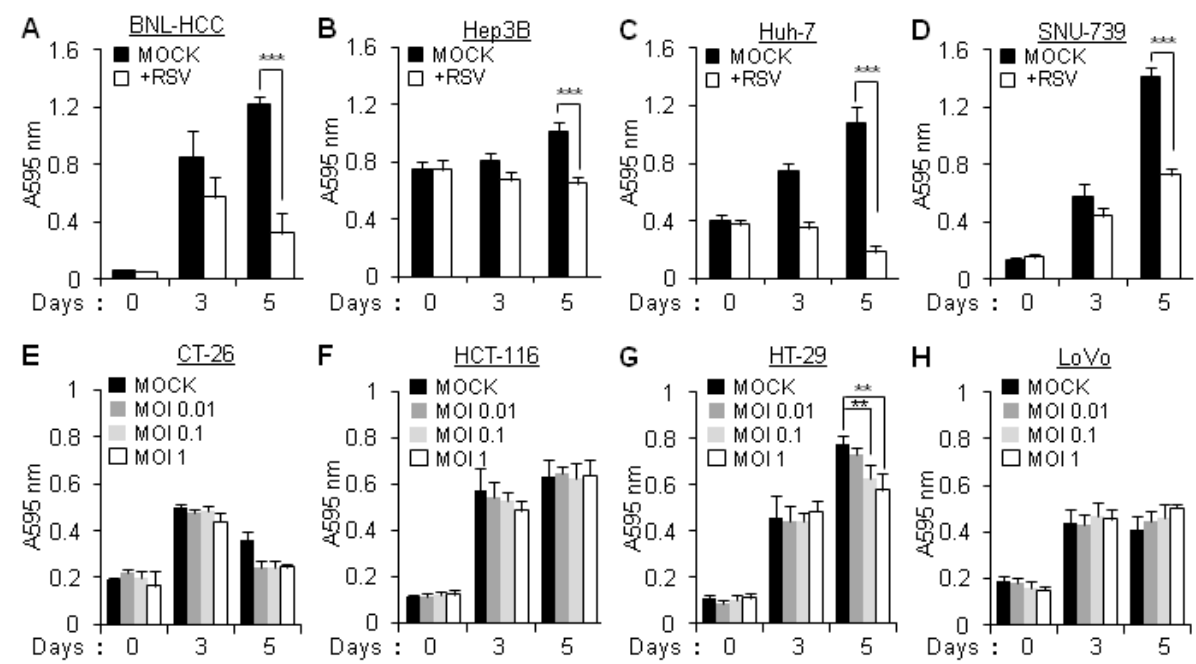

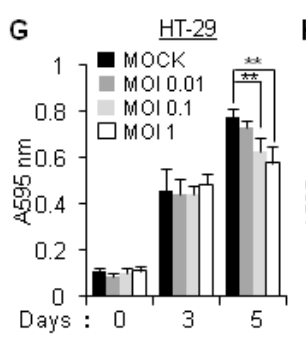

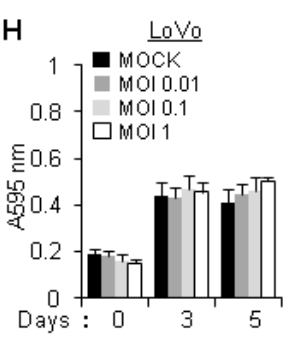

Fig. 1. Effects of RSV infection on the growth of cancer cell lines. HCC cell lines (A-D) and colon cancer cell lines (E-H) were cultured for $24 \mathrm{~h}$ and then infected with RSV $(0.1 \mathrm{MOI}$ for HCC cell lines, 0.01-1 MOI for colon cancer cell lines) for five days. Cell growth was measured by MTT assay. Each bar represents Mean \pm SD values obtained from three individual experiments. This experiment was performed three times with similar results. Student's $t$ test $\mathrm{P}$ values for the RSV infection versus con-

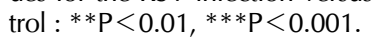



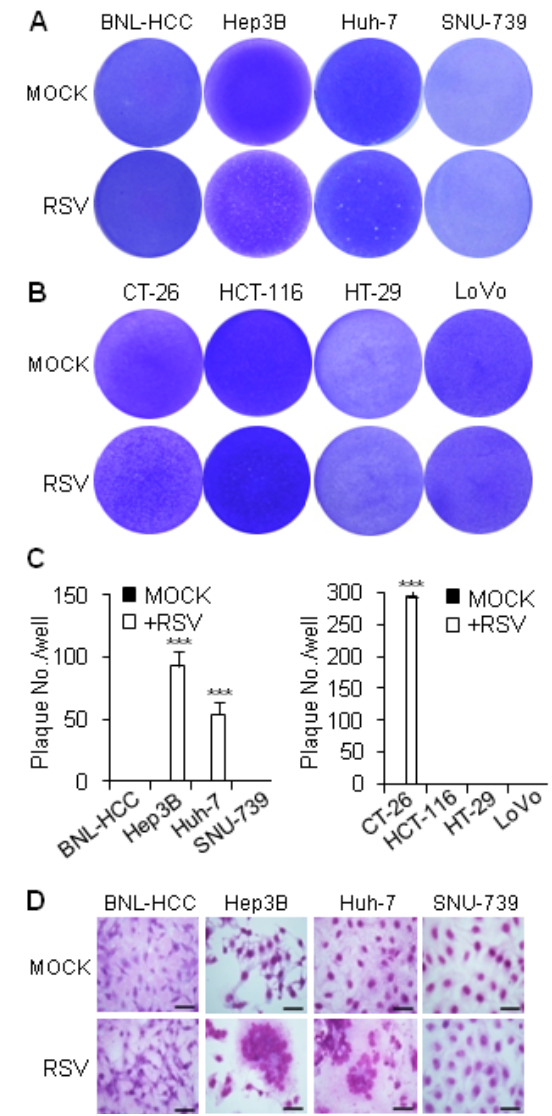

Fig. 2. Identification of RSV infection and syncytial formation in the cancer cell lines. (A and B) Plaque formation by RSV infection. HCC cell lines (A) and colon cancer cell lines (B) were cultured in 12-well plates for $12 \mathrm{~h}$ and then infected with RSV for $2 \mathrm{~h}$. The cell culture plates were coated with $0.3 \%$ immunodiffusion grade agar, and then incubated until plaque formation. After 5-7 days, the plates were stained with crystal violet solution after removal of agar. (C) The number of plaques was counted and compared. Each bar represents Mean \pm SD value obtained from three individual experiments. This experiment was performed three times with similar results. Student's $t$ test $P$ values for the RSV infection versus control : ***P<0.001. (D) Syncytial formation induced by RSV infection. HCC cell lines (BNL-HCC, Hep3B cells, Huh-7 and SNU-739 cells) were cultured on the cover slips in 12-well plates for $12 \mathrm{~h}$. The cells were infected with RSV. After 5-7 days, the cells were fixed and then stained with Giemsa staining solution. Scale bars: $20 \mu \mathrm{m}$. This experiment was performed three times with similar results.

cells (data not shown).

Cell cycle regulation in BNL-HCC cells after RSV infection To determine the effects of RSV infection on the cell cycle, DNA content was analyzed in RSV-infected BNL-HCC cells through staining with propidium iodide, followed by flow cytometry analysis. It was difficult to analyze the cell cycle of Hep3B and Huh-7 cells because the cells formed syncytia after RSV infection. The percentage of G0/G1 phase in the RSV-infected

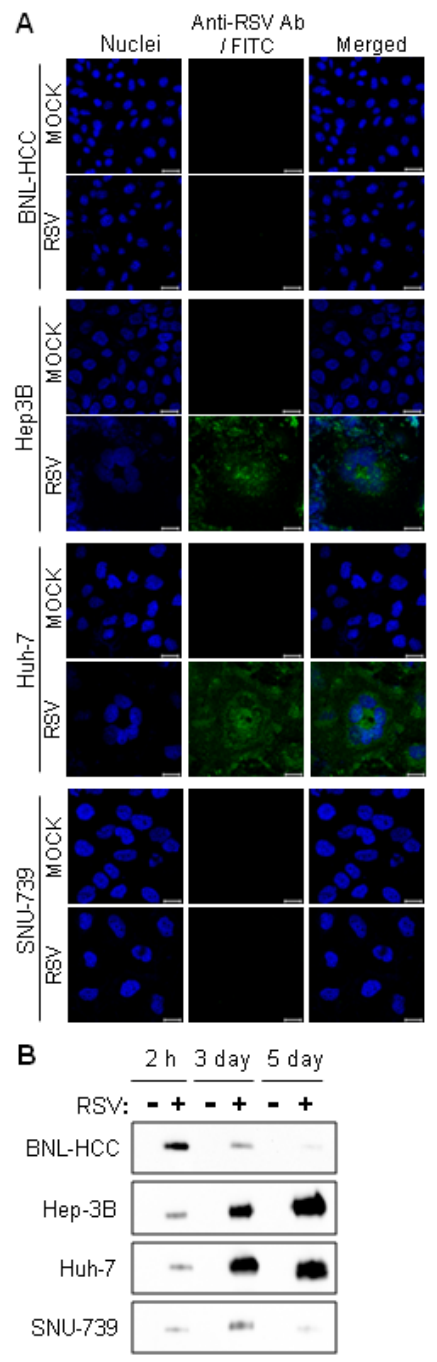

Fig. 3. Identification of RSV growth in the cancer cell lines. HCC cell lines (BNL-HCC, Hep3B cells, Huh-7 and SNU-739) were cultured for $12 \mathrm{~h}$ on the cover slips in 12-well plates. The cells were infected with RSV and cultured for 72 h. (A) Growth of RSV in the cell lines were measured by goat anti-RSV antibody and anti-goat Alexa Flour 488 with confocal microscopy. The nuclei were stained with Hoechst 33258. Scale bars: $10 \mu \mathrm{m}$. (B) The cell were harvested and expression of RSV protein was determined by Western blot analysis using a monoclonal antibody to anti-RSV G protein.

BNL-HCC cells was significantly higher than in the mock control. However, the percentage of S-phase and G2/M-phase in the RSV-infected BNL-HCC cells was significantly lower than in the control (Supplementary Fig. 1). These experiments suggest that G0/G1 cell-cycle-arrest was induced by RSV infection in BNL-HCC cells. 

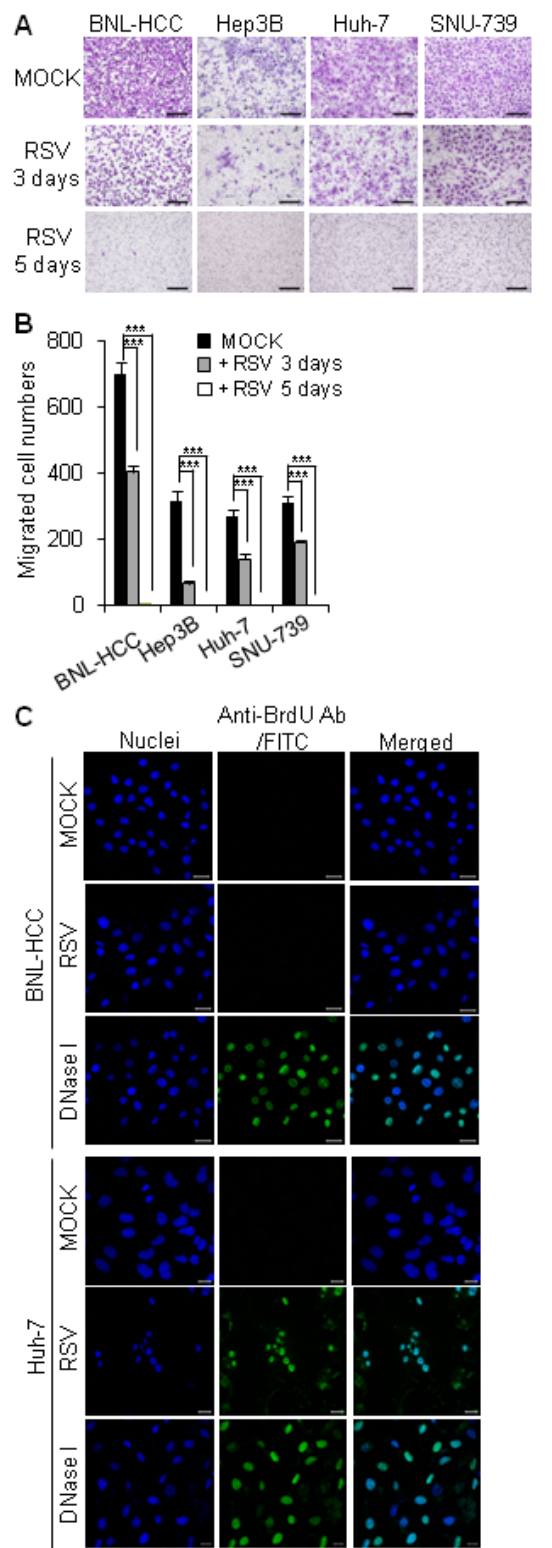

Fig. 4. Effect of RSV infection on cell migration and apoptosis. (A) Effect of RSV infection on cell migration. HCC cell lines (BNL-HCC, Hep3B cells, Huh-7 and SNU-739) were infected with RSV and the RSV-infected cells ( 3 days or 5 days) were placed on top of the trans-well chamber. After incubation for $12 \mathrm{~h}$ or 36 $h$, the cells that had migrated to the lower sides of the membranes were counted. Scale bars: $50 \mu \mathrm{m}$. (B) The number of migrated cells was counted and compared. Each bar represents Mean \pm SD values obtained from three individual experiments. This experiment was performed three times with similar results. Student's $t$ test $\mathrm{P}$ values for the RSV infection versus control : ***P $<0.001$. (C) Effect of RSV infection on apoptosis. BNL-HCC and SNU-739 cells were infected with RSV in 12-well plates. After incubation for $96 \mathrm{~h}$, the cells were fixed and then stained with DeadEnd ${ }^{\mathrm{TM}}$ Fluorometric TUNEL system. The nuclei were stained with Hoechst 33258. Scale bars: $10 \mu \mathrm{m}$.

\section{DISCUSSION}

Oncolytic virus is a potential new anticancer therapeutic with the potential to inhibit the growth of cancer cells and to induce apoptosis. Therefore, many investigators have reported relevant results and are making greater efforts to develop the oncolytic viruses as anti-cancer therapeutics $(22,23)$. The oncolytic effects of RSV were observed in lung, skin, and prostate cancer cells previously (19-21). The RSV infection rate was significantly enhanced in cancer cells compared to non-cancerous cells. The enhanced RSV infection led the cancer cells to virus-induced apoptosis, whereas the non-cancerous cells were unaffected (24-28). Based on the results obtained from prostate cancer model, viral production and suppressive activity of RSV in cancer cells are related with deregulated activation of NF- $\mathrm{KB}$ pathway or defect of antiviral cytokine IFN $\alpha / \beta$-induced STAT-1 activation $(19,20)$. However, the effects of RSV in HCC cells have not yet been investigated. Therefore, we searched for oncolytic effects of RSV in HCC cell lines.

The cell growth was significantly decreased by RSV infection in HCC cell lines such as BNL-HCC, Hep3B, Huh-7 and SNU-739 (Fig. 1). However, cell growth in other HCC cell lines (SNU-761 and SNU-423 cells) and colon-cancer cell lines (CT-26, HCT-116, HT-29 and LoVo cells) was not affected by RSV infection or merely affected by RSV at the high MOI. Therefore, the effect of RSV infection on cancer cell growth seems to vary depending on the type of cell infected. Further investigation of the effects of RSV infection on cell growth in many types of cancer cells, may provide more information for application of oncolytic RSV therapeutics.

Plaque formation and syncytial formation was induced by RSV infection in Hep3B and Huh-7 cells along with growth suppression (Fig. 1 and 2). RSV protein expression was also detected in Hep3B and Huh-7 cells (Fig. 3). However, only Huh-7 cells showed apoptosis after RSV infection (Fig. 4). Furthermore, inhibition of cell migration by RSV infection was observed in BNL-HCC, Hep3B, Huh-7 and SNU-739 cells. Interestingly, the growth of BNL-HCC and SNU-739 cells is suppressed by RSV without prominent viral replication. When we checked expression of RSV protein by Western blot analysis, low level of protein expression was detected in BNL-HCC and SNU-739 cells. Therefore, we speculate that low level of RSV proteins induced by RSV infection can inhibit the growth of the cells. Prominent viral production and syncytial formation can be an additional feature in other cells. Therefore, the relationship between RSV infection and HCC cell growth is not clear yet and it's necessary to further investigate the effects of RSV infection in a variety of HCC cells.

In short, this report demonstrates that the RSV inhibits cell growth and induces apoptosis in a few HCC cell lines. Our results provide evidence that the RSV has potential as a tool for oncolytic virotherapy in HCC. Further investigations are needed to understand the molecular mechanisms involved in the RSV-mediated oncolytic effects, and to apply the RSV in potential oncolytic therapeutics for HCC. 


\section{MATERIALS AND METHODS}

\section{MTT assay}

To measure the effect of the RSV on the growth of HCC and colon cancer cells, an MTT assay was performed with a 3-(4,5 dimethylthiazole-2-yl)-2,5-diphenyltetrazolium bromide (MTT, Sigma-Aldrich, ST. Louis, MO, USA) solution as described previously $(29,30)$. The cells were seeded in 48-well plates at a density of $1 \times 10^{4}$ cells/well (BNL-HCC, Hep3B, CT-26, HCT-116 and LoVo) or $5 \times 10^{3}$ cells/well (Huh-7, SNU-739, HT-29). After $24 \mathrm{~h}$ cell culture, the cells were infected with RSV at a MOI of 0.1 and incubated at $37^{\circ} \mathrm{C}$ in a $5 \% \mathrm{CO}_{2}$ incubator for three and five days. After incubation, the supernatant was removed and $100 \mu$ of MTT solution (2 $\mathrm{mg} / \mathrm{ml}$ MTT in autoclaved distilled water) was added to each well. The plates were incubated for $4 \mathrm{~h}$ at $37^{\circ} \mathrm{C}$, and the culture media were removed. Then $100 \mu$ of DMSO was added for solubilization of the formazan crystals. The color development was analyzed at $595 \mathrm{~nm}$ by a Spectra Max $250 \mathrm{mi}-$ croplate reader (Molecular Devices, Sunnyvale, CA, USA).

\section{Plaque assay in cancer cell lines}

Cells were grown in 12-well plates starting with $1 \times 10^{6}$ cells/ well (BNL-HCC, Hep3B, CT-26, HCT-116 and LoVo) or $5 \times 10^{5}$ cells/well (Huh-7, SNU-739, HT-29) for $12 \mathrm{~h}$. The cells were infected with RSV (MOI of 0.1) and incubated for $2 \mathrm{~h}$ with repeated rocking every $15-20 \mathrm{~min}$. The media were removed and $2 \mathrm{ml}$ of F12/DMEM containing $2 \mathrm{mM}$ L-glutamine, $4 \%$ BSA, $10 \mathrm{mM}$ HEPES, $2.4 \%$ sodium bicarbonate, $100 \mathrm{U} / \mathrm{ml}$ penicillin, 100 $\mathrm{mg} / \mathrm{ml}$ streptomycin, and $0.3 \%$ immuno-diffusion grade agar was added. The plates were then incubated until plaque formation. After 5-7 days, the plates were fixed with $0.5 \%$ paraformaldehyde in PBS and incubated at room temperature for 12-24 h. The agar was removed using flowing tap water and each well stained with $1 \mathrm{ml}$ of crystal violet solution. After $6 \mathrm{~h}$, the staining solutions were washed away with flowing tap water. The plaque formation was photographed with a Nikon camera (Nikon, Tokyo, Japan).

\section{Giemsa staining}

BNL-HCC, Hep3B cells, Huh-7, and SNU-739 cells were cultured on the cover slips in 12-well plates stating with $1 \times 10^{4}$ cells/well (BNL-HCC and Hep3B) or $5 \times 10^{3}$ cells/well (Huh-7 and SNU739) for $12 \mathrm{~h}$. The cells were infected with RSV at a MOI of 0.1, and incubated until cytopathic effect was observed. The plates were rinsed with PBS, fixed with $100 \% \mathrm{MeOH}$ at $25^{\circ} \mathrm{C}$ for $5 \mathrm{~min}$, washed with PBS, and then dried for $4 \mathrm{~h}$. The cells were stained with $1 \mathrm{ml}$ of Giemsa stain solution (Sigma-Aldrich) at $25^{\circ} \mathrm{C}$ for $2 \mathrm{~h}$, and mounted on slide-glasses with a mounting solution fluoromount-G (Southern Biotech, Birmingham, Alabama, USA). The cells were scanned with a light microscope (E-200, Nikon).

\section{Immunofluorescence staining and confocal microscopy} BNL-HCC, Hep3B cells, Huh-7 and SNU-739 cells were cultured for $12 \mathrm{~h}$ on the cover slips in 12-well plates starting with $2 \times 10^{4}$ cells/well (BNL-HCC and Hep3B) or $1 \times 10^{4}$ cells/ well (Huh-7 and SNU-739). The cells were infected with RSV at a MOI of 0.1, and incubated for $72 \mathrm{~h}$. After incubation, the supernatant was removed and the cells were fixed with $4 \%$ paraformaldehyde, permeabilized, and blocked with PBS containing 3\% BSA and $0.1 \%$ Triton-X 100 (PBS-BT). The samples were stained with the goat anti-RSV antibody (Gene Way Biotech, San Diego, CA, USA) in PBS-BT ( $10 \mu \mathrm{g} /$ each sample) for $2 \mathrm{~h}$. The samples were then treated with anti-goat Alexa Flour 488 (Life Technologies) for $1 \mathrm{~h}$ (1:500). The nuclei were stained with Hoechst 33258 (Life Technologies, 1:2000) for $5 \mathrm{~min}$. The samples were rinsed with PBS-BT three times, and mounted on slide-glasses with a mounting solution (fluoromount-G, Southern Biotech). The florescence was scanned with an LSM 710 laser scanning microscope (Carl Zeiss, Jena, Germany) at the Cooperative Center for Research Facilities, Hallym University, Chuncheon, Korea.

\section{Western blot analysis}

BNL-HCC, Hep3B cells, Huh-7 and SNU-739 cells were cultured for $12 \mathrm{~h}$ on the cover slips in 12-well plates and infected with RSV for $72 \mathrm{~h}$ as described in "Immunofluorescence staining and confocal microscopy". The cells were harvested and resolved by SDS-PAGE in $12.5 \%$ reducing polyacrylamide gels. The separated proteins were electro-transferred to a nitrocellulose membrane (Bio-Rad, Hercules, CA, USA) and the blot was analyzed by immunoblotting with a monoclonal antibody to G protein of RSV (31).

\section{Migration assay}

Migration assays were performed with trans-well chambers with $8-\mu \mathrm{m}$ porosity (32). The lower side of the trans-well chamber membranes was coated with gelatin $(10 \mathrm{mg} /$ well). BNL-HCC, Hep3B cells, Huh-7 and SNU-739 cells were cultured in $150 \mathrm{~mm}$ dishes starting with $1 \times 10^{5}$ cells/dish (BNL-HCC and Hep3B) or $5 \times 10^{4}$ cells/dish (Huh-7 and SNU-739). The cells were infected with RSV at a MOI of 0.1 , and incubated for $2 \mathrm{~h}, 72 \mathrm{~h}$ and $120 \mathrm{~h}$. The RSV-infected cells were placed on top of the trans-well chamber. DMEM (BNL-HCC), EMEM (Hep3B), and RPMI (Huh-7 and SNU-739) medium containing 10\% FBS was placed in the lower chamber. After incubation for $12 \mathrm{~h}$ (BNL-HCC, Huh-7 and SNU-739) or $36 \mathrm{~h}$ (Hep3B), the cells that migrated to the lower surface of the filters were fixed, stained with crystal violet, and counted under a light microscope (E-200, Nikon).

\section{TUNEL assay}

Terminal deoxynucleotidyl transferase (TdT)-mediated dUTP nick-end labeling (TUNEL) assay was performed in accordance with manufacturer specifications (33). BNL-HCC, Hep3B cells, Huh-7 and SNU-739 cells were cultured for $12 \mathrm{~h}$ on the cover slips in 12-well plates starting with $1 \times 104$ cells/well (BNL- HCC and Hep3B) or $5 \times 10^{3}$ cells/well (Huh-7 and SNU-739). The cells were infected with RSV at a MOI of 0.1 , and incubated for $96 \mathrm{~h}$. The cells were fixed with $4 \%$ paraformaldehyde and permeabilized with PBS-T. The DeadEnd ${ }^{\mathrm{TM}}$ Fluorometric TUNEL system (Promega, Madison, WI, USA) was used for nick-end la- 
beling with TdT. The nuclei were stained with Hoechst 33258 (Life Technologies, 1:2,000) for $10 \mathrm{~min}$. The samples were washed with PBST and PBS, and then mounted on slide-glasses with a mounting solution Fluoromount-G (Southern Biotech). The florescence was scanned with an LSM 710 (Carl Zeiss). The DNase I treatment served as a control for TUNEL-positive cells.

\section{SUPPLEMENTARY DATA}

Supplementary Data are available at BMB Reports online: Supplementary MATERIALS AND METHODS, Supplementary Fig. 1.

\section{ACKNOWLEDGEMENTS}

This research was supported by the National Research Foundation of Korea (NRF) funded by the Ministry of Science, ICT \&Future Planning in the Republic of Korea (2013R1A2A2A03067981, 2014M3C1A3051473, 2009-0093812).

\section{REFERENCES}

1. Parkin DM, Bray F, Ferlay J and Pisani $P$ (2005) Global cancer statistics, 2002. CA Cancer J Clin 55, 74-108

2. Forver A, Llivet JM and Bruix (2012) Hepatocellular carcinoma. Lancet 379, 1245-1255

3. El-Serag HB and Rudolph KL (2007) Hepatocellular carcinoma: epidemiology and molecular carcinogenesis. Gastroenterology $132,2557-2576$

4. Hoofnagle JH (2004) Hepatocellular carcinoma: summary and recommendations. Gastroenterology 127, S319-S323

5. Perz JF, Armstrong GL, Farrington LA, Hutin YJ and Bell BP (2006) The contributions of hepatitis B virus and hepatitis C virus infections to cirrhosis and primary liver cancer worldwide. J Hepatol 45, 529-538

6. Douek M and Taylor I (2003) Good practice and quality assurance in surgical oncology. Lancet Oncol 4, 626-630

7. Llovet JM, Burroughs A and Bruix J (2003) Hepatocellular carcinoma. Lancet 362, 1907-1917

8. Avila MA, Berasain C, Sangro B and Prieto J (2006) New therapies for hepatocellular carcinoma. Oncogene 25, 3866-3884

9. Woller N, Gürlevik E, Ureche Cl, Schumacher A and Kühnel F (2014) Oncolytic viruses as anticancer vaccines. Front Oncol 4, 188

10. Russell SJ, Peng KW and Bell JC (2012) Oncolytic virotherapy. Nat Biotechnol 30, 658-670

11. Liu TC, Hwang T, Park BH, Bell J and Kirn DH (2008) The targeted oncolytic poxvirus JX-594 demonstrates antitumoral, antivascular, and anti-HBV activities in patients with hepatocellular carcinoma. Mol Ther 16, 1637-1642

12. Kim JH, Oh JY, Park BH et al (2006) Systemic Armed Oncolytic and Immunologic Therapy for Cancer with JX-594, a Targeted Poxvirus Expressing GM-CSF. Mol Ther 14, 361-370

13. Heo J, Breitbach CJ, Moon A et al (2011) Sequential therapy with JX-594, a targeted oncolytic poxvirus, followed by sorafenib in hepatocellular carcinoma: preclinical and clinical demonstration of combination efficacy. Mol Ther 19, 1170-1179
14. Heo J, Reid T, Ruo L et al (2013) Randomized dose-finding clinical trial of oncolytic immunotherapeutic vaccinia JX-594 in liver cancer. Nat Med 19, 329-336

15. Chang J (2011) Current progress on development of respiratory syncytial virus vaccine. BMB Rep 44, 232-237

16. Atreya PL and Kulkarni S (1999) Respiratory syncytial virus strain A2 is resistant to the antiviral effects of type I interferons and human MxA. Virology 261, 227-241

17. Tregoning JS and Schwarze J (2010) Respiratory viral infections in infants: causes, clinical symptoms, virology, and immunology. Clin Microbiol Rev 23, 74-98

18. Falsey AR and Walsh EE (2000) Respiratory syncytial virus infection in adults.Clin Microbiol Rev 13, 371-384

19. Echchgadda I, Kota S, DeLa Cruz I et al (2009) Anticancer oncolytic activity of respiratory syncytial virus. Cancer Gene Ther 16, 923-935

20. Echchgadda I, Chang TH, Sabbah A et al (2011) Oncolytic targeting of androgen-sensitive prostate tumor by the respiratory syncytial virus (RSV): consequences of deficient interferon-dependent antiviral defense. BMC Cancer 11, 43

21. Salimi V, Tavakoli-Yaraki M, Mahmoodi M et al (2013) The Oncolytic Effect of Respiratory Syncytial Virus (RSV) in Human Skin Cancer Cell Line, A431. Iran Red Crescent Med J 15, 62-67

22. Kelly E and Russell SJ (2007) History of Oncolytic Viruses: Genesis to Genetic Engineering. Mol Ther 15, 651-659

23. Kirn D, Martuza RL, Zwiebel J (2001) Replication-selective virotherapy for cancer: Biological principles, risk management and future directions. Nat Med 7, 781-787

24. Bell JC (2007) Oncolytic viruses: what's next? Curr Cancer Drug Targets 7, 127-131

25. Stanford MM and McFadden G (2007) Myxoma virus and oncolytic virotherapy: a new biologic weapon in the war against cancer. Expert Opin Biol Ther 7, 1415-1425

26. Mohr I (2005) To replicate or not to replicate: achieving selective oncolytic virus replication in cancer cells through translational control. Oncogene 24, 7697-7709

27. Everts B and van der Poel HG (2005) Replication-selective oncolytic viruses in the treatment of cancer. Cancer Gene Ther $12,141-161$

28. Parato KA, Senger D, Forsyth PA and Bell JC (2005) Recent progress in the battle between oncolytic viruses and tumours. Nat Rev Cancer 5, 965-976

29. Kim HR, Heo YM, Jeong Kl et al (2012) FGF-2 inhibits TNF- $\alpha$ mediated apoptosis through upregulation of $\mathrm{BCl} 2-\mathrm{A} 1$ and Bcl-xL in ATDC5 cells. BMB Rep 45, 287-292

30. Youn JK, Kim DW, Kim ST et al (2014) PEP-1-HO-1 prevents MPTP-induced degeneration of dopaminergic neurons in a Parkinson's disease mouse model. BMB Rep 47, 569-574

31. Kim S and Chang J (2012) Baculovirus-based Vaccine Displaying Respiratory Syncytial Virus Glycoprotein Induces Protective Immunity against RSV Infection without VaccineEnhanced Disease. Immune Netw 12, 8-17

32. Lee YD, Cui MN, Yoon HH, Kim HY, Oh IH and Lee JH (2014) Down-modulation of Bis reduces the invasive ability of glioma cells induced by TPA, through NF-kB mediated activation of MMP-9. BMB Rep 47, 262-267

33. Kim DS, Sohn EJ, Kim DW et al (2012) PEP-1-p18 prevents neuronal cell death by inhibiting oxidative stress and Bax expression. BMB Rep 45, 532-537 